\title{
Editorial
}

\section{IWP auf dem Weg in die Zukunft}

DOI 10.1515/iwp-2014-0059

Das wissenschaftliche Publikationswesen ist im Umbruch. Autorinnen und Autoren stellen ihre Manuskripte heute in digitaler Form bereit. Immer mehr Leserinnen und Leser nutzen Laptop, Tablet oder Smartphone, um Informationen $\mathrm{zu}$ rezipieren. Hochschulen, außeruniversitäre Forschungseinrichtungen Bibliotheken und Verlage reagieren in unterschiedlicher Weise. Gedruckte Zeitschriftenabonnements werden von umfassenden Lizenzverträgen für den Online-Zugriff auf elektronische Medien und Pay-perview-Angebote abgelöst. Patron-driven acquisition überträgt die Verantwortung für den Ankauf von Verlagsprodukten und den Bestandsaufbau von Bibliotheken auf die Benutzer. Verlage bieten maßgeschneiderte individuelle Profildienste, um auf Neuerscheinungen aufmerksam zu machen. Bibliotheken und Verlage lernen die Vorlieben ihrer Benutzerinnen und Kunden so immer besser kennen.

Auch De Gruyter Saur, der Verlag der IWP, bietet Dienstle an, die von der DGI als Herausgeberin der Zeitschrift bereits 2014 genutzt wurden, künftig aber noch stärker im Bewusstsein der Mitglieder verankert und von ihnen in Anspruch genommen werden sollen.

\section{eTOC Alert}

Für alle Mitglieder der DGI und die Abonnenten ist die IWP zwei bis drei Wochen vor Auslieferung des gedruckten Heftes bereits online auf dem Verlagsserver verfügbar. Auch die Bibliotheken, die die IWP lizenziert haben, stellen ihrer Klientel die aktuelle IWP so bereits vor Einarbeitung der gedruckten Ausgabe online bereit.

Wer informiert werden will, wann das neue Heft zur Verfügung steht, kann sich auf der Website von De Gruyter registrieren, um den eToc Alert zu beziehen, und so über den Inhalt des neuen Heftes informiert $\mathrm{zu}$ werden. Bisher haben nach Aussage des Verlags nur sehr wenige Mitglieder diesen unentgeltlichen Dienst abonniert.

\section{Ahead-of-Print}

Außerdem gibt es die Möglichkeit der Publikation von einzelnen Aufsätzen „Ahead of Print“. Dies bedeutet, dass Beiträge, sobald sie positiv begutachtet und seitens Redaktion und Verlag fertig gestellt sind, sofort einzeln online publiziert werden können, ohne dass sie bereits einem bestimm- ten Zeitschriftenheft zugeordnet und mit Seitenzahlen versehen worden sind. Auch davon haben wir bisher nur einmal Gebrauch machen können, weil die meisten Autorinnen und Autoren gewohnt sind, auf einen bestimmten Redaktionsschlusstermin hinzuarbeiten und bisher sehr selten einmal Manuskripte im Stehsatz bleiben mussten.

\section{Vier gedruckte Ausgaben 2015}

DGI und Verlag haben sich darauf verständigt, dass ab 2015 die Publikationsform Ahead-of-print ausgebaut werden soll und nur noch vier gedruckte Hefte der IWP erscheinen sollen. Aufsätze und Berichte stehen dann online den Leserinnen und Lesern schnell zur Verfügung und sind durch den DOI zitierbar. Mit der Konzentration auf vier Ausgaben kommen wir aber auch dem Wunsch von Mitgliedern und Bibliotheken nach, die sich eine geringere Erscheinungsfrequenz der gedruckten IWP wünschen. Die Informationsmenge wird nicht abnehmen, der gewohnte Seitenumfang eines Jahrgangs bleibt erhalten.

\section{Zusammenarbeit mit dem HI}

Nachdem der Versuch gescheitert ist, die DFG zur Förderung einer europäischen Open-Access-Zeitschrift für die Informationswissenschaft $\mathrm{zu}$ gewinnen, hat die DGI ab 2015 den Schulterschluss mit dem Hochschulverband Informationswissenschaft vereinbart. Der wissenschaftliche Teil der IWP soll grundsätzlich von Gastherausgebern aus dem Kreis der Mitglieder des HI betreut werden, die zusammen mit den Gutachtern die wissenschaftliche Qualität der Beiträge sicherstellen.

Die Informationspraxis soll jedoch nicht zu kurz kommen; aktuelle Fragestellungen und gründliche Darstellungen aus dem Tagesgeschäft der Informationspraktiker sind der Redaktion weiterhin stets willkommen.

Die DGI als Herausgeberin der IWP und die Redaktion hoffen, mit dieser Weiterentwicklung der IWP auf breite Zustimmung der Mitglieder und Abonnenten zu stoßen. Bitte nutzen Sie den Hinweisdienst eTOC-Alert, um sofort von neu online gestellten IWP-Ausgaben zu erfahren und teilen Sie der Geschäftsstelle, dem Vorstand oder der Redaktion mit, wie Sie sich die Weiterentwicklung der IWP in Zukunft wünschen.

Marlies Ockenfeld 